\title{
Relações entre os Testes de Bender e Matrizes Progressivas Coloridas de Raven na Avaliação da Inteligência
}

\author{
Fermino Fernandes Sisto \\ Daniel Bartholomeu \\ Fabián Javier Marin Rueda \\ Acácia Aparecida Angeli dos Santos \\ Ana Paula Porto Noronha \\ Universidade São Francisco
}

\begin{abstract}
RESUMO
Esta pesquisa estudou as relações entre o Teste Gestaltico Visomotor de Bender avaliado conforme o Sistema de Pontuação Gradual (B-SPG) e as Matrizes Progressivas Coloridas de Raven. Participaram desta pesquisa 280 sujeitos com idades variando de sete a 10 anos com média de oito anos (DP=1,11) de ambos os sexos. Os testes foram aplicados coletivamente. Dentre os resultados, encontraram-se correlações negativas e significativas entre a medida do Bender e das séries do Raven, bem como com seu total, indicando que quanto mais distorções as crianças fazem nas cópias, menor a pontuação no Raven. A análise de grupos extremos formados com base no desempenho nas Matrizes Progressivas corrobora esse achado, na medida em que demonstrou diferenças das crianças mais e menos inteligentes quanto à distorção das figuras, sendo que as mais inteligentes apresentaram menos distorções no Bender em comparação com as que tinham pior desempenho no Raven.
\end{abstract}

Palavras-chave: avaliação da inteligência; Bender - Sistema de Pontuação Gradual; Matrizes Progressivas Coloridas de Raven; validação.

\section{ABSTRACT \\ Relationship Between Bender and Raven's Colored Progressive Matrices in Intelligence Assessment}

This research studied the relationships between the Bender Visomotor Gestalt Test measured according to the Sistema de Pontuação Gradual (B-SPG) and the Raven's Colored Progressive Matrices. A total of 280 male and female subjects aged from seven to 10 years (Mean = eight yearsold, DP $=1,11)$. The tests were collectively applied. Among the results, negative and significant correlations were observed among the Bender and the series of Raven measures, as well as with its total, indicating that the more distortions the children made in the copies, the lower the scores of the Raven Test. The contrasting groups analysis based on the Progressive Matrices corroborated these data since it demonstrated differences among the higher and lower intelligent children in the distortion of the figures. The most intelligent children presented fewer distortions in Bender contrasted to the ones who had worse performance in the Raven.

Keywords: intelligence assessment; Bender - Sistema de Pontuação Gradual; Raven's Colored Progressive Matrices; validity.

Como medida de avaliação o Teste de Bender foi utilizado com finalidade clínica quando de sua publicação em 1938. Os protocolos eram analisados qualitativamente, pois não havia um sistema de correção para a atribuição de uma pontuação para cada um dos nove desenhos que constam do instrumento (Sisto, Noronha \& Santos, 2004; entre outros). Com base na obtenção de bons resultados diagnósticos com o teste, ele passou a ser amplamente utilizado, surgindo a preocupação com uma maior objetividade na análise dos protocolos (Machado, 1978; Sisto, Bueno \& Rueda, 2003; Sisto, Santos \& Noronha, 2004). Em razão disso, foram propostos vários sistemas de avaliação e interpretação das figuras (Hutt, 1969; Lacks, 1984; Posada, 2002; por exemplo). 
Independentemente do critério adotado na avaliação dos protocolos dos sujeitos, sabe-se que ele tem se prestado a diferentes fins. Em uma perspectiva mais ampla, procura avaliar a percepção e reprodução das figuras com base no pressuposto de que esses processos são determinados por princípios biológicos e de ação sensório-motriz. Assim, variam de acordo com o padrão de desenvolvimento e o estado patológico funcional e orgânico do indivíduo. Em decorrência, há estudos que visam verificar as características neurológicas em diferentes grupos com dificuldades específicas, dentre as quais se encontram estudos com pacientes epilépticos e esquizofrênicos (Lee \& Oh, 1998; Maciel Jr. \& La Puente, 1983; entre outros), crianças com deficiência auditiva (Cariola, Piva, Yamada \& Bevilacqua, 2000; Gemignani \& Chiari, 2000) e pacientes portadores da síndrome de imunodeficiência adquirida (Mattos, 1991).

A literatura sobre os sistemas de avaliação cognitiva do Bender fornece estudos que procuraram relacionar seus escores com a inteligência. De acordo com Koppitz (1987), o Teste Gestáltico pode ser utilizado para avaliar a inteligência em crianças durante o período de amadurecimento da percepção visomotora. Nesse sentido, a autora realizou um estudo para verificar se havia correlação entre os testes de inteligência, Escala de Inteligência de Stanfor-Binet (Forma L), Wechsler Intelligence Scale for Children (WISC), e o Bender. Participaram 239 crianças de cinco a 10 anos com problemas emocionais ou de aprendizagem, divididas em dois grupos conforme o QI; em um, o escore médio era de 98 e no outro de 63. Utilizando a análise de correlação produto-momento de Pearson entre os escores do Bender e dos testes de inteligência em cada nível de idade, foram obtidos todos os índices de correlação negativos e significativos. Com base nesse resultado, a autora concluiu que o Teste Gestáltico pode ser utilizado como medida indicativa da inteligência das crianças.

Também Baer e Gale (1967) avaliaram as diferenças e relações entre a habilidade visomotora e o nível de inteligência em crianças institucionalizadas e não institucionalizadas de primeira série, por meio do teste de Bender (Sistema Koppitz) e do Teste de Maturidade Mental Califórnia. Dentre os resultados, verificaram que crianças institucionalizadas tinham menor habilidade em comparação com as demais. Entretanto, notaram que ao serem retiradas as contribuições da medida de inteligência, por meio de uma análise de covariância, a diferença do Teste Gestáltico entre os grupos desaparecia. Os autores encontraram ainda, correla- ções entre as medidas fornecidas pelos instrumentos para o grupo de crianças institucionalizadas e não para as outras.

McNamara, Porterfield e Miller (1969) detectaram as relações entre a Escala de Inteligência Primária Pré-escolar de Wechsler (WPPSI), o Teste de Matrizes Progressivas Coloridas de Raven (CPM) e o Teste de Bender. No geral, os resultados indicaram que o teste Gestáltico está mais associado a áreas de performance do WPPSI e pode ser mais apropriado para obter estimativas de QI de pré-escolares do que o CPM.

Ainda o estudo de Koppitz (1987) foi realizado a fim de verificar se o Bender manteria relação com os subtestes do WISC. Os sujeitos foram 90 alunos com idades entre seis a 11 anos, divididos em dois grupos, sendo um de primeiras e segundas séries e outro de terceiras e quartas séries, cujo QI médio era de 95. Foram administrados o WISC e o Bender e os resultados sugeriram que o QI total do WISC está mais relacionado ao desempenho no Bender nas terceiras e quartas séries.

Por sua vez, Silberberg e Feldt (1968) investigaram configurações psicométricas no WISC e Bender para crianças de primeiras séries com problemas de leitura. Examinando os dados concluíram que o escore padrão do WISC, bem como do Teste Gestáltico de Bender (sistema Koppitz) não acrescentaram informações relevantes para a análise da performance de crianças com atrasos de leitura.

As relações entre os escores da Escala Wechsler e os escores do Teste Gestáltico de Bender foram estudadas por Doubros e Mascarenhas (1969) em 71 crianças de seis a 12 anos. Índices de correlação negativos e significativos foram detectados entre os dois testes, com coeficientes que variaram entre $r=-0,22$ e $r=-0,43$, não havendo diferenças de gênero.

Também Vance, Fuller e Lester (1986) estudaram o desempenho de 33 crianças no Bender, no Minnesota Perceptual Diagnostic Test (MPDT-R) e no Wide Range Achievement Test (WRAT) para averiguar se teriam caráter preditivo do desempenho no WISC-R. Os resultados evidenciaram que tanto o MPDT-R quanto o WRAT demonstraram superioridade em relação ao Bender na predição do QI tal como fornecido pelo WISC-R.

A seu turno, Yousefi, Shahim, Razavieh e Mehryar (1992) forneceram dados normativos para o Teste de Bender pelo Sistema de avaliação de Koppitz para crianças iranianas, cuja validade foi estabelecida pela 
diferenciação por idade e por correlação com o DFH (Sistema de Goodenough-Harris). Os resultados mostraram boas associações entre os instrumentos e permitiram aos autores a conclusão de que o Bender poderia ser empregado para avaliar o desenvolvimento cognitivo-intelectual das crianças.

Em uma pesquisa brasileira, Rueda, Bartholomeu e Sisto (2004) analisaram as relações entre o Bender segundo os critérios de Koppitz e o Teste do Desenho da Figura Humana (DFH) de Goodenough em 312 crianças do ensino fundamental. Dentre os resultados, encontraram correlações negativas e significativas entre as medidas de distorção e integração, bem como do total do Bender e o DFH separadamente por sexo e idade (de sete a dez anos) e com a amostra toda (os coeficientes que apresentaram significância estatística variaram de $-0,16$ a $-0,38)$. A tendência encontrada indicou que o bom desempenho das crianças no DFH denotou menos problemas na cópia das Figuras do Bender. Esses dados conduziram os autores a sugerirem elementos comuns entre os testes, indicando uma relação entre os constructos mensurados.

Na literatura, o sistema de correção mais utilizado é o Developmental Bender Test Scoring System, criado por Koppitz em 1963, e destinado à avaliação da maturidade neurológica da criança para o diagnóstico de lesão cerebral (Machado, 1978). Seu sistema teve como pressuposto que o desenvolvimento das funções gestálticas visomotoras seria fruto da maturação. Nesse sistema somente as irregularidades grosseiras identificadas no desenho das crianças são pontuadas, não sendo considerados pequenos desvios. Nesse sentido, pontuações altas indicam um desempenho pobre, enquanto pontuações baixas são qualificadas como um bom desempenho.

No Brasil também foi o sistema mais usado, embora os sistemas de Santucci e Galifret-Granjon (1968) e Santucci e Pêcheux (1981) tenham sido amplamente utilizados. Quanto ao sistema de Koppitz, por não possuir um manual com normas e estudos psicométricos brasileiros, não foi recomendado pelo Conselho Federal de Psicologia. Além disso, muitos estudos evidenciaram críticas ao sistema Koppitz de correção do Bender (Rueda, Bartholomeu \& Sisto, 2004; Pinelli Jr., 1990; Sisto, Noronha \& Santos, 2004; entre outros).

Também Kroeff $(1988,1992)$ estudou o método de correção de Koppitz, fornecendo normas específicas para escolas brasileiras públicas e privadas. $\mathrm{O}$ autor verificou diferenças nos resultados em razão das séries escolares e das idades, não demonstrando, todavia, quais dessas são de fato discriminadas, sendo essa uma restrição encontrada nesse trabalho.

Valendo-se das críticas apresentadas pela literatura, principalmente no que se refere aos critérios de correção, Sisto, Noronha e Santos (2005) propuseram o Bender-Sistema de Pontuação Gradual (B-SPG) como alternativa de aplicação e de correção das figuras do teste. Diferentemente do sistema de Koppitz, que considera apenas a presença ou ausência de quatro critérios de avaliação, quais sejam, a distorção de forma, a integração, a rotação e a perseveração, o B-SPG somente avalia a distorção de forma em um sistema que pontua os erros na cópia das figuras de acordo com a gravidade do erro. Também, enquanto o primeiro sistema avalia em uma escala de 30 pontos, o B-SPG possui uma escala de 21 pontos.

Sisto, Noronha e Santos (2005) mostraram a sensibilidade do sistema para diferenciar mudanças desenvolvimentais, apresentando evidência de validade de construto para crianças de seis a 10 anos. O manual do B-SPG apresenta ainda estudos que comprovam a unidimensionalidade da escala, sugerindo que os indicadores da maturação visomotora mensuram, de fato, uma única dimensão. Ao lado disso, utilizando o B-SPG, Suehiro e Santos (2005) desenvolveram um estudo de validade de critério para o sistema, apontando-o como capaz de discriminar crianças com dificuldades de aprendizagem daquelas que não as tem.

Apesar das evidências de validade desse sistema, julgou-se interessante estudar suas relações com inteligência, mais especificamente com o Teste Matrizes Progressivas Coloridas de Raven (Angelini, Alves, Custódio, Duarte \& Duarte, 1999). De certa forma, procurou-se averiguar se ele manteria as mesmas relações com inteligência que a literatura tem registrado em relação ao sistema de Koppitz.

\section{MÉTODO}

\section{Participantes}

Participaram desta pesquisa 239 sujeitos com idades variando de sete a 10 anos com uma média de idade de oito anos $(\mathrm{DP}=1,11)$. Todos cursavam de primeira a quarta séries do ensino fundamental de uma escola municipal do interior do estado de São Paulo, sendo que $55,2 \%$ eram meninas e $44,8 \%$ meninos. As crianças foram selecionadas verificando-se se a série que cursavam era correspondente à sua idade. Desse modo, nesse estudo não foram incluídas crianças repetentes ou com defasagem de série. 


\section{INSTRUMENTOS}

\section{A - Teste Gestáltico Visomotor de Bender - Sistema de Pontuação Gradual (B-SPG)}

Consiste de nove figuras (A, 1, 2, 3, 4, 5, 6, 7 e 8) que são apresentadas uma a uma, para serem copiadas pelo sujeito em uma folha em branco, sem auxílio mecânico. O sistema de avaliação dos aspectos maturacionais adotado foi desenvolvido por Sisto, Noronha e Santos (2005), que tem como características principais (a) considerar apenas o aspecto de distorção de forma nas cópias; (b) atribuir a cada item uma pontuação que pode variar de um a três pontos, conforme a gravidade do erro. No caso de não haver desvios relativos à distorção da forma, atribui-se a pontuação zero. As figuras A, 1, 2, 3, 2, 5, 7a, 7b e 8 possuem pontuações que variam de zero a dois pontos. Já a figura 6 é avaliada de zero a três pontos. Desse modo, a pontuação total de distorção de forma que uma criança pode obter nesse instrumento é de 21 pontos.

\section{B - Teste Matrizes Progressivas Coloridas de Raven}

$\mathrm{O}$ instrumento consiste de um caderno que possui, em cada folha, um desenho com um pedaço faltante que o sujeito é solicitado a completar, devendo escolher entre seis alternativas a que ele acredita que o preenche corretamente, havendo somente uma alternativa correta. $O$ instrumento é subdividido em três séries $\mathrm{A}, \mathrm{Ab}$ e $\mathrm{B}$, com níveis distintos de dificuldade e nos 12 itens de cada uma, perfazendo 36 itens para o teste como um todo. Faz-se a avaliação do teste atribuindo-se um ponto a cada resposta correta. A pontuação total dos indivíduos corresponde ao número de acertos e pode chegar a 36 pontos. A composição dos escores para as séries $\mathrm{A}, \mathrm{Ab}$ e $\mathrm{B}$ resulta da pontuação dos itens de cada uma delas, sendo que o total de pontos que se pode atingir é de 12 .

Sua validade de construto foi obtida pela diferenciação das idades cronológicas em relação às médias no teste o que, conforme Anastasi (1988), pode ser considerado como evidência de validade (Angelini, Alves,
Custódio, Duarte \& Duarte, 1999). Ao lado disso, apresentou uma correlação item-total entre 0,30 e 0,80 em todos os itens, menos nos itens A1, A2, A3, A4, $\mathrm{A} 5, \mathrm{Ab} 1$ e B1. A consistência interna foi satisfatória, sendo também utilizada como estimativa da validade de construto. A precisão do instrumento foi calculada pelo método das metades e as correlações entre as partes, corrigidas pela fórmula de Spearman-Brown, forneceram coeficientes para o sexo masculino de 0,92 , para o feminino de 0,90 e na amostra total de 0,92 .

\section{PROCEDIMENTO}

Para a aplicação do B-SPG foi entregue lápis e borracha, além de uma folha de papel sulfite A4. Nesse estudo, as transparências com as figuras do Teste Gestáltico foram apresentadas no quadro com o auxílio de um retroprojetor, conforme instruções para aplicação coletiva do Manual do B-SPG. Assim, foi explicado às crianças que nove figuras seriam projetadas uma a uma no quadro e que elas deveriam copiá-las, da melhor maneira, numa mesma folha em branco. Quando as crianças terminavam a cópia de uma figura, projetava-se a seguinte.

Em seguida, foram distribuídos os cadernos do Raven e uma folha de respostas do teste. A aplicação deste instrumento seguiu minuciosamente a proposta em seu manual.

\section{RESULTADOS}

Os resultados serão apresentados em três blocos. No primeiro deles, estão as estatísticas descritivas para ambos os instrumentos; no segundo encontram-se as relações estabelecidas entre as informações desses dois grupos de dados; finalmente, verificou-se em que medida os grupos extremos em relação às séries do Raven e de sua pontuação total são discriminados também na pontuação do Bender.

Foram calculadas as médias, os desvios-padrão e as pontuações mínima e máxima para cada série do Raven e para o Bender-SPG. Os resultados obtidos estão apresentados na Tabela 1.

TABELA 1

Estatísticas Descritivas dos Testes de Raven e B-SPG ( $N=280)$

\begin{tabular}{cccccc}
\hline & SERIE A & SERIE Ab & SERIE B & Raven & B-SPG \\
\hline Média & 8,78 & 7,72 & 5,85 & 22,35 & 9,13 \\
Desvio Padrão & 1,98 & 2,92 & 2,92 & 6,84 & 3,89 \\
Mínimo & 3,00 & 0,00 & 0,00 & 5,00 & 2,00 \\
Máximo & 12,00 & 12,00 & 12,00 & 35,00 & 20,00 \\
\hline
\end{tabular}


As crianças apresentaram um melhor desempenho na série $\mathrm{A}$ em relação às séries $\mathrm{Ab}$ e $\mathrm{B}$, uma vez que a pontuação máxima que se poderia atingir seria 12 pontos e a média foi de 8,88 . Na série $\mathrm{Ab}$, a média das pontuações diminuiu $(7,85)$, o que, de fato é esperado considerando que a dificuldade dos itens é maior nessa série. Todavia, ainda ficou acima do ponto médio dessa subescala. Na série B identificou-se novamente uma diminuição da média de acertos $(5,98)$, valor um pouco menor que o ponto médio dessa subescala. $\mathrm{O}$ escore médio do total do Raven, entretanto, foi acima do ponto médio da escala, indicando que para os alunos, de forma geral, o instrumento não foi muito difícil. Outro dado interessante é que algumas crianças obtiveram pontuação de 35 no teste, conseguindo resolver corretamente a quase totalidade dos itens. Outras ainda acertaram todos os itens nas diferentes séries, embora não se tratasse das mesmas crianças em todas as séries.

Em relação ao teste de Bender, a média das crianças ficou abaixo do ponto médio da escala, sugerindo que elas, de modo geral, não apresentaram muitas distorções em seus desenhos, embora algumas crianças tenham obtido 20 dos 21 pontos possíveis. Levando-se em conta a pontuação média total obtida, podese dizer que esse instrumento foi aparentemente fácil para a maioria das crianças, embora algumas tenham feito distorcidamente a maioria das figuras.

\section{Relações entre o B-SPG e o Teste Matrizes Progressivas Coloridas de Raven}

Com vistas a verificar até que ponto as medidas fornecidas pelos instrumentos em estudo estariam associadas, correlacionou-se tais escores por meio da prova de correlação Produto-Momento de Pearson, estabelecendo o nível de significância de 0,05. Também se estabeleceu coeficientes de correlação parciais entre as medidas com controle da idade, para retirar seu efeito dos coeficientes. Os resultados dessa análise estão na Tabela 2.

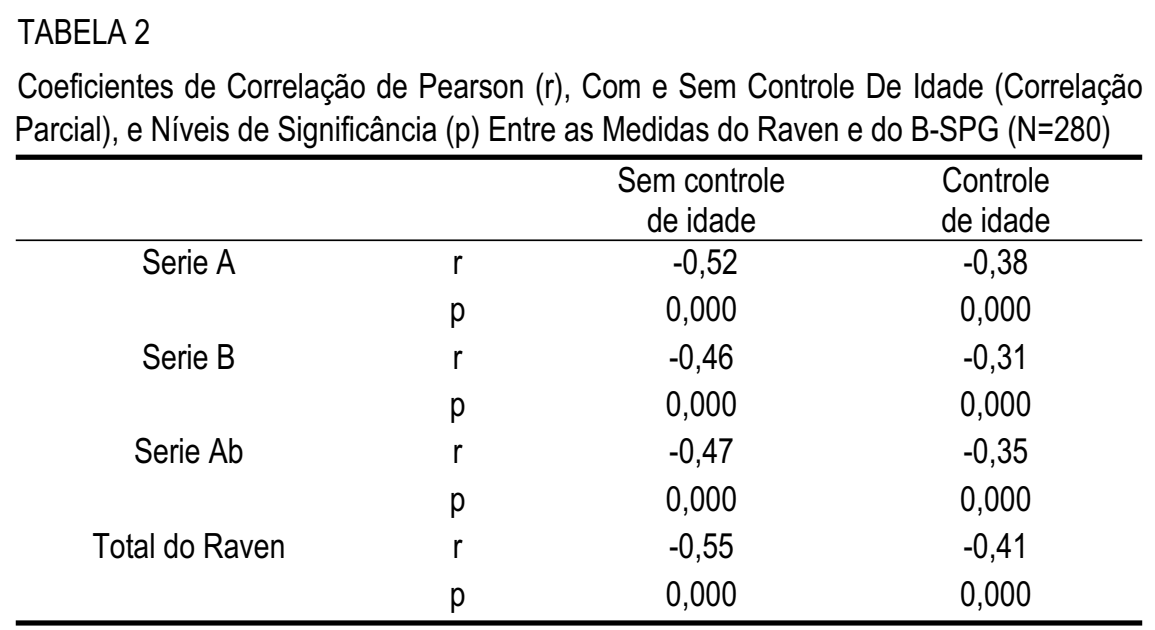

Verificou-se que todas as medidas em questão forneceram correlações significativas e negativas, controlando ou não o efeito da idade sobre as pontuações. Aparentemente, o controle de tal efeito apenas diminuiu um pouco a magnitude do coeficiente de correlação sem que, contudo, pudesse mudar a interpretação. Ou seja, a tendência das correlações foi mantida.

Assim, por estes dados, observou-se que as medidas de distorção do Bender estiveram mais fortemente imbricadas com as medidas de inteligência. Considerando que as altas pontuações nas medidas do Bender indicam uma maior distorção e que escores altos no Teste Matrizes Progressivas indicam melhor desempenho, as tendências observadas demonstram que as crianças mais inteligentes evidenciam menos distorções ao copiarem as figuras do Teste Gestáltico. É interessante observar ainda que os coeficientes entre as medidas do Bender e as séries do Raven estiveram mais fortemente associadas com a série A do que com a $\mathrm{Ab}$ e B.

Procurou-se ainda averiguar, em cada série escolar, até que ponto as medidas desses instrumentos estariam associadas, como pode ser visto na Tabela 3. Por estes dados, pôde-se verificar que, diferentemente das outras séries, na primeira série os coeficientes obtidos entre todas as medidas tomadas não foram significativos. 
TABELA 3

Coeficientes de Correlação de Pearson Entre as Medidas de Distorção do Teste de Bender e das Matrizes Progressivas de Raven Por Série Escolar ( $\mathrm{N}=280)$

\begin{tabular}{cccccc}
\hline \multicolumn{2}{c}{ Medidas do Raven / séries } & Serie A & Série Ab & Série B & Total do Raven \\
\hline 1a série & $\mathrm{r}$ & $-0,24$ & $-0,31$ & $-0,15$ & $-0,29$ \\
$\mathrm{~N}=78$ & $\mathrm{p}$ & 0,036 & 0,005 & 0,177 & 0,010 \\
2 a série & $\mathrm{r}$ & $-0,31$ & $-0,36$ & $-0,19$ & 0,35 \\
$\mathrm{~N}=59$ & $\mathrm{p}$ & 0,016 & 0,005 & 0,145 & 0,007 \\
3a série & $\mathrm{r}$ & $-0,38$ & $-0,29$ & $-0,37$ & $-0,40$ \\
N=85 & $\mathrm{p}$ & 0,000 & 0,008 & 0,000 & 0,000 \\
4 a série & $\mathrm{r}$ & $-0,48$ & $-0,26$ & $-0,37$ & $-0,41$ \\
N=58 & $\mathrm{p}$ & 0,000 & 0,052 & 0,004 & 0,001 \\
\hline
\end{tabular}

A série A do teste de Raven manteve correlações significativas com a medida de distorção em todas as séries escolares; ao passo que a série $\mathrm{Ab}$ associou-se significativamente com esse escore do Bender na primeira, segunda e terceira séries. Por sua vez, a série B correlacionou-se significativamente nas terceira $\mathrm{e}$ quarta séries. Finalmente, a pontuação total do Raven apresentou índices significativos, e todos negativos, em todas as séries. As correlações significativas variaram de $-0,24$ a $-0,48$. Dos 16 coeficientes possíveis, 13 configuraram-se como significativos e negativos.

\section{Diferenciação dos grupos extremos nas medidas do Raven por meio dos escores do Bender}

Para verificar se os grupos extremos constituídos com base na pontuação total do Raven discriminariam as medidas do Bender, utilizou-se a prova $t$ de Student, adotando nível de significância de 0,05. O Grupo 1 englobou $25 \%$ dos participantes com as menores pontuações e o grupo 2 , os $25 \%$ que obtiveram as maiores pontuações. Os resultados dessa análise encontram-se na Tabela 4.

TABELA 4

Comparação, Valores de t e $\mathrm{p}$ das Medidas do Bender nos Grupos Extremos do Total do Raven ( $\mathrm{N}=280)$

\begin{tabular}{ccccccc}
\hline & $\begin{array}{c}\text { Grupos } \\
\text { extremos do } \\
\text { Raven }\end{array}$ & N & Média & Desvio Padrão & $t$ & $p$ \\
\hline Figura A & 1 & 83 & 0,57 & 0,70 & 4,73 & 0,000 \\
Figura 1 & 2 & 62 & 0,11 & 0,32 & & \\
Figura 2 & 1 & 83 & 1,14 & 0,99 & 3,53 & 0,001 \\
& 2 & 62 & 0,58 & 0,90 & & \\
Figura 3 & 1 & 83 & 1,29 & 0,57 & 4,21 & 0,000 \\
& 2 & 62 & 0,87 & 0,61 & & \\
Figura 2 & 1 & 83 & 1,73 & 0,47 & 4,44 & 0,000 \\
& 2 & 62 & 1,29 & 0,73 & & \\
Figura 5 & 1 & 83 & 1,25 & 0,64 & 5,07 & 0,000 \\
& 2 & 62 & 0,74 & 0,54 & & \\
Figura 6 & 1 & 83 & 0,93 & 0,60 & 1,61 & 0,111 \\
& 2 & 62 & 0,77 & 0,53 & & \\
Figura 7a & 1 & 83 & 1,47 & 0,83 & 5,52 & 0,000 \\
& 2 & 62 & 0,79 & 0,58 & & \\
Figura 7b & 1 & 83 & 1,22 & 0,80 & 7,07 & 0,000 \\
& 2 & 62 & 0,37 & 0,58 & & \\
Figura 8 & 1 & 83 & 1,08 & 0,86 & 5,72 & 0,000 \\
& 2 & 62 & 0,37 & 0,55 & & \\
Total Bender & 1 & 83 & 0,88 & 0,85 & 6,59 & 0,000 \\
& 2 & 62 & 0,13 & 0,34 & & \\
& 1 & 83 & 11,57 & 3,82 & 10,08 & 0,000 \\
\hline
\end{tabular}


Verificou-se que somente a medida de distorção da Figura 5 não diferenciou os grupos. As demais pontuações discriminaram os grupos extremos formados por meio das pontuações do Raven, sendo que as médias dos escores do Bender do grupo de pior desempenho no Raven foram maiores que as do de melhor performance, indicando um maior número de irregularidades destas crianças nas cópias dos desenhos. Em outros

TABELA 5

Comparação da Medida do Bender nos Grupos Extremos das Séries do Raven ( $\mathrm{N}=280)$

\begin{tabular}{lccccc}
\hline & $\begin{array}{c}\text { Grupos extremos } \\
\text { das séries do Raven }\end{array}$ & Média & $\begin{array}{c}\text { Desvio } \\
\text { Padrão }\end{array}$ & $\mathrm{t}$ & $\mathrm{p}$ \\
\hline Série $\mathrm{A}$ & 1 & 11,55 & 3,89 & 10,21 & 0,00 \\
& 2 & 5,85 & 2,20 & & \\
Série $\mathrm{Ab}$ & 1 & 11,35 & 3,91 & 7,76 & 0,00 \\
& 2 & 6,70 & 2,50 & & \\
Série B & 1 & 10,98 & 3,78 & 8,56 & 0,00 \\
& 2 & 6,18 & 2,41 & & \\
\hline
\end{tabular}

\section{DISCUSSÃO}

Este estudo foi proposto, por um lado, tendo por base a concepção de inteligência de Spearman que, por meio da análise fatorial, inferiu a existência de um fator geral na capacidade mental humana, consubstanciado no Matrizes Progressivas Coloridas de Raven (Angelini, Alves, Custódio, Duarte \& Duarte, 1999). Esse fator seria constituído pela capacidade edutiva, compreendida pela edução de relações e correlatos, entre as diversas áreas do conhecimento, empregada na resolução de tarefas.

Por outro lado, a literatura tem apontado uma relação entre a inteligência e os escores do Bender sistematicamente (Koppitz, 1987; Baer \& Gale, 1967, Doubros \& Mascarenhas, 1969, entre outros). Considerando que o Sistema de Pontuação Gradual, desenvolvido por Sisto, Noronha e Santos (2006) é bastante recente, perguntou-se até que ponto essa associação relatada pela literatura se manteria nesse novo sistema para a avaliação dos protocolos do Teste de Bender. Nesse contexto, esta pesquisa se propôs a investigar as relações das pontuações do Sistema de Pontuação Gradual do Teste de Bender com as do teste Matrizes Progressivas Coloridas de Raven.

As análises iniciais revelaram que a medida fornecida pelo Sistema de Pontuação Gradual do Teste de Bender correlacionou-se negativa e significativamente com as do Teste de Raven, tanto no que tange às séries em específico, quanto no que concerne à pontuação geral. Assim, quanto mais capacidade edutiva as crianças evidenciaram, menos foram observadas distorções no Teste Gestáltico. termos, o pior desempenho no Raven corresponde a figuras mais distorcidas no Bender.

Foi ainda estudada a medida do Bender em relação aos grupos extremos constituídos por meio das pontuações das séries A, Ab e B do Raven, usando os mesmos critérios para a formação dos grupos anteriores. Os dados da Tabela 5 indicaram que o total do Bender discriminou os grupos extremos em razão das séries $\mathrm{A}, \mathrm{Ab}$ e B.

As análises por série escolar, separadamente, indicaram que, na primeira série, essas medidas não estavam associadas. Na segunda série, somente a série B não forneceu correlações significativas com o Bender. $\mathrm{Na}$ terceira série todas as medidas efetuadas correlacionaram-se e a magnitude dos coeficientes foi maior nessa série do que nas outras; e, finalmente, na quarta série, somente a série $A b$ não se correlacionou significativamente com o Bender. Entretanto, pouco mais de $60 \%$ dos coeficientes possíveis mostraram-se significativos. Essas diferenças nos coeficientes entre as séries devem ser pensadas com base na maturidade visomotora e a inteligência das crianças. Pode-se sugerir que há momentos no desenvolvimento em que essas variáveis aparecem mais fortemente relacionadas, enquanto que em outros, não. Nesse sentido, parece que apesar de suficientemente paralelos, a ponto de se correlacionarem, eles parecem ser independentes. Duas são as razões para isso: uma delas se refere ao fato de que houve flutuações nos resultados encontrados; e a outra se relaciona ao fato de que, mesmo sendo significativa, uma boa parte da variância (a maior parte dela) não foi explicada pela associação encontrada, sugerindo a presença de outros fatores nessa relação.

Os coeficientes de correlação obtidos por Doubros e Mascarenhas (1969) entre a Escala Wechsler de inteligência e o Teste de Bender, conforme o sistema de Koppitz, variaram entre $-0,22$ e $-0,43$. Nesses termos, os resultados desta pesquisa mostraram-se promissores, uma vez que os coeficientes variaram de $-0,47$ a $-0,55$ (ou $-0,43$ e $-0,52$, com controle de idade), 
sugerindo uma magnitude de correlação mais intensa. Deve-se considerar, entretanto, que os pressupostos de inteligência dos testes com os quais o Bender foi comparado são distintos, embora ambos se refiram a capacidades intelectuais de crianças. Estes resultados contrapõem-se aos encontrados por McNamara e colaboradores (1969) que sugeriram que o teste de Bender estaria mais associado às Escalas Wechsler do que com as Matrizes Progressivas de Raven, ainda que, vale ressaltar, tenham sido utilizados sistemas de pontuações distintos para o Bender.

No estudo de Koppitz (1987), a autora procurou verificar até que ponto o Bender se relacionava com os subtestes do WISC, separando os sujeitos em dois grupos, um de primeiras e segundas e outro de terceiras e quartas séries. Sugeriu-se que o QI das crianças de terceiras e quartas séries relacionou-se mais aos escores do Teste Gestáltico. Esse dado é em parte corroborado pela presente pesquisa, uma vez que, para as crianças de segunda série as correlações entre as medidas do total do Bender e do Raven foram também significativas, mas os maiores coeficientes foram estabelecidos nas terceiras e quartas séries. Entretanto, considerando que as séries no estudo de Koppitz foram agrupadas, é possível interpretar que a primeira série tenha influenciado no resultado.

Verificou-se ainda, comparando-se os grupos extremos em cada série em particular do Raven bem como com seu total, que os grupos contrastantes discriminaram as medidas de cada figura do Bender, com exceção da Figura 5. Assim, essa análise permitiu afirmar que as crianças que apresentaram as melhores pontuações, seja no total do Raven, seja em suas séries em específico, tenderam a apresentar menos distorções no Bender em comparação com as crianças que obtiveram os piores desempenhos nas avaliações do Raven.

Poder-se-ia questionar se o desenvolvimento não estaria produzindo essa associação encontrada. Apesar de que em um teste é exigida uma cópia e em outro, raciocínio, as informações foram referentes a crianças que estavam em desenvolvimento. No entanto, ao se remover o efeito da idade sobre os coeficientes, a magnitude dos coeficientes foi muito pouco afetada, sugerindo que o desenvolvimento, aferido por meio da idade, não seria suficiente para explicar as correlações encontradas.

\section{CONSIDERAÇÕES FINAIS}

Assim, em síntese, esse conjunto de resultados aqui obtidos sugere que o Teste Gestáltico de Bender, corrigido pelo Sistema de Pontuação Gradual, pode ser utilizado para fornecer uma estimativa da habilidade intelectual de crianças de segunda a quarta séries. Os dados desta pesquisa podem ser considerados como evidência de validade para os critérios de Sisto, Noronha e Santos (2005).

Algumas considerações devem, todavia, ser feitas quanto às limitações desse trabalho. Embora os coeficientes de correlação encontrados entre os instrumentos em questão tenham sido satisfatórios, o estudo de Sisto, Rueda e Bartholomeu (2006), analisando a unidimensionalidade do Teste Matrizes Progressivas Coloridas de Raven, revelou que ele estaria mensurando mais de uma dimensão, pondo em dúvida se estaria avaliando, de fato, o fator g de Spearman, tal como consta de seu Manual.

Nesse contexto, a interpretação das correlações encontradas neste trabalho pode ser questionada. Em outros termos, seria interessante investigar quais outras dimensões o Teste Raven estaria medindo e quais mecanismos psicológicos subjazem a elas, pois poderiam fornecer mais informações sobre o que o B-SPG poderia estar medindo. Este fato convida a novas pesquisas que busquem também a validade do Sistema de Pontuação Gradual em relação à inteligência com outros instrumentos e critérios diferentes.

\section{REFERÊNCIAS}

Anastasi, A. (1988). Psychological testing (6 ${ }^{\mathrm{a}}$ ed.). New York: Macmillan Publishing Co.

Angelini, A. L., Alves, I. C. B., Custódio, E. M., Duarte, W. F., \& Duarte, J. L. M. (1999). Manual: Matrizes Progressivas Coloridas de Raven. São Paulo. Centro Editor de Testes e Pesquisas em Psicologia.

Baer, D. J., \& Gale, R. A. (1967). Intelligence and Bender Gestalt test performance of institutional and noninstitutional school children. Journal of Genetic Psychology, 111(1), 119-124.

Cariola, T. C., Piva, R. A., Yamada, M. O., \& Bevilacqua, M. C. (2000). A prova gráfica de organização perceptiva para crianças de quatro a seis anos deficientes auditivas. Pediatria Moderna, 36(9), 588-594.

Doubros, S. G., \& Mascarenhas, J. (1969). Relations among Wechsler full-scale scores, Organicity-sensitive Subtest scores and Bender-Gestalt errors scores. Perceptual and Motor Skills, 29(3), 719-722.

Gemignani, E. Y. M. C., \& Chiari, B. M. (2000). Escala de Maturação do Teste de Bender em um grupo de crianças deficientes auditivas. Pró-fono, 12(2), 49-53.

Hutt, M. L. (1969). The Hutt adaptation of the Bender Gestalt test. New York: Grune \& Stratton.

Koppitz, E. (1987). O Teste Gestáltico de Bender para crianças (R. N. Piccoli, Trad.) Porto Alegre: Artes Médicas.

Kroeff, P. (1988). Normas brasileiras para o Teste de Bender. Psicologia: Reflexão e Crítica, 1/2(3), 12-19.

Kroeff, P. (1992). Desempenho de crianças no Teste de Bender e nível sócio-econômico-cultural. Psicologia: Reflexão e Crítica, 5(2), 119-126. 
Lacks, P. (1984). Bender-Gestalt screening for brain disfunction. New York: John Hiley.

Lee, S. Y., \& Oh, S. W. (1998). Visuoperceptual and constructive ability disturbances of patients with traumatic brain injury in Hutt Adaptation of the Bender Gestalt Test. Korean Journal of Clinical Psychology, 17(1), 311-317.

Machado, M. C. L. (1978). Uso do teste de Bender para avaliar a organização perceptivo-motora de escolares paulistas. Dissertação de mestrado não publicada, Pontifícia Universidade Católica de São Paulo.

Maciel, Jr., J. A., \& La Puente, M. (1983). Avaliação multimodal do teste de Bender no psicodiagnóstico da epilepsia. Revista Brasileira de Neurologia, 2(19), 55-58.

Mattos, P. (1991). Os distúrbios mentais orgânicos e a síndrome de imunodeficiência adquirida. Jornal Brasileiro de Psiquiatria, 40(7), 375-381.

McNamara, J. R., Porterfield, C. L., \& Miller, L. E. (1969). The relationship of the Wechsler Preschool and Primary Scale of Intelligence with the Coloured Progressive Matrices (1956) and the Bender Gestalt Test. Journal of Clinical Psychology, 25(1), 65-68.

Pinelli Jr., B. (1990). Adaptação do "Teste do Desenvolvimento da Integração Visomotora (VMI)” para uso no Brasil. Dissertação de mestrado não publicada, Universidade de Brasília.

Posada, M. C. (2002). Un sistema alternativo para la evaluacion del Test Gestaltico Visomotor de Bender. Revista Iberoamericana de Diagnostico y Evaluación-Psicologica, 14(2), 9-31.

Rueda, F. R. M., Bartholomeu, D., \& Sisto, F. F. (2004) Desenho da Figura Humana e Teste de Bender: Um estudo correlacional. Revista Psicologia Argumento, 23(39), 39-44.

Santucci, H., \& Galifret-Granjon, N. (1968). Prova Gráfica de Organização Perceptiva. Em R. Zazzo (Org.), Manual para o exame psicológico da criança (pp. 233-268). São Paulo: Mestre Jou.

Santucci, H., \& Pêcheux, M. G. (1981). Prova Gráfica de Organização Perceptiva para crianças de 6 a 14 anos. Em R. Zazzo
(Org.), Manual para o exame psicológico da criança (pp. 291438). São Paulo: Mestre Jou.

Silberberg, N., \& Feldt, L. S. (1968). Intellectual and perceptual correlates of reading difficulties. Journal of School Psychology, 6(4), 237-245.

Sisto, F. F., Bueno, J. M. H., \& Rueda, F. J. M. (2003). Traços de personalidade na infância e distorção e integração de formas: Um estudo de validade. Psicologia em Estudo, 8(1), 77-84.

Sisto, F. F., Noronha, A. P. P., \& Santos, A. A. A. (2004). Distorção de forma no Teste de Bender: Questionando seu critério de validade. Revista do Departamento de Psicologia da UFF, 16(2), 139-154.

Sisto, F. F., Noronha, A. P. P., \& Santos, A. A. A. (2005). Bender - Sistema de Pontuação Gradual B-SPG. São Paulo: Vetor.

Sisto, F. F., Rueda, F. J. M., \& Bartholomeu, D. (2006). Estudo sobre a unidimensionalidade do Teste Matrizes Progressivas Coloridas de Raven. Psicologia: Reflexão e Crítica, 19(1), 6673.

Sisto, F. F., Santos, A. A. A., \& Noronha, A. P. P. (2004). Critério de integração do Teste de Bender: Explorando evidências de validade. Avaliação Psicológica, 3(1), 13-20.

Suehiro, A. C. B., \& Santos, A. A. A. (2005). O Bender: Sistema de Pontuação Gradual como indicativo de dificuldades de aprendizagem. Avaliação Psicológica, 4(1) 23-32.

Vance, B., Fuller, G. B., \& Lester, M. L. (1986). A comparison of the Minnesota Perceptual Diagnostic Test Revised and the Bender Gestalt. Journal of Learning Disabilities, 19(4), 211214.

Yousefi, F., Shahim, S., Razavieh, A., \& Mehryar, A. H. (1992). Some normative data on the Bender Gestalt Test performance of iranian children. British Journal of Educational Psychology, $62(3), 410-416$.

\section{Sobre os autores:}

Fermino Fernandes Sisto: Doutor pela Universidad Complutense de Madrid, Livre - docente pela Unicamp e docente do curso de Psicologia e do Programa de Estudos Pós-graduados em Psicologia, da Universidade São Francisco. Bolsista produtividade em pesquisa do CNPq.

Daniel Bartholomeu: Psicólogo, mestrando do Programa de Pós-Graduação Stricto Sensu em Psicologia da Universidade São Francisco e bolsista da CAPES.

Fabián Javier Marín Rueda: Psicólogo, doutorando do Programa de Pós-Graduação Stricto Sensu em Psicologia da Universidade São Francisco e bolsista da CAPES.

Acácia Aparecida Angeli dos Santos: Doutora em Psicologia Escolar e Desenvolvimento Humano pela Universidade de São Paulo. Docente da graduação e do Programa de Pós-Graduação Stricto Sensu da Universidade São Francisco. Bolsista produtividade em pesquisa do CNPq.

Ana Paula Porto Noronha: Doutora em Psicologia: Ciência e Profissão pela PUC-Campinas. Docente da graduação e do Programa de Pós-Graduação Stricto Sensu da Universidade São Francisco. Bolsista produtividade em pesquisa do CNPq.

Endereço para correspondência: daniel.bartholomeu@unisal.am.br; d_bartholomeu@yahoo.com.br. 